\title{
Comparison of efficacy of extracorporeal magnetic innervation and Kegel exercises for stress urinary incontinence in adult women: study protocol for a randomized controlled trial
}

\author{
Mislav Mikuš ${ }^{1}$, Kristina Fišter ${ }^{2}$, Bernarda Škegro ${ }^{3}$, Giovanni Buzzaccarini ${ }^{4}$, Marco Noventa $^{4}$, \\ Antonio Simone Laganá ${ }^{5}$, Slavko Orešković ${ }^{1}$, Mario Ćorić ${ }^{1}$, Držislav Kalafatić ${ }^{1}$, Vladimir Banović ${ }^{1}$ \\ ${ }^{1}$ Department of Obstetrics and Gynecology, University Hospital Center Zagreb, Zagreb, Croatia \\ ${ }^{2}$ School of Medicine, University of Zagreb, Zagreb, Croatia \\ ${ }^{3}$ Sestre milosrdnice, University Hospital Centre, Zagreb, Zagreb, Croatia \\ ${ }^{4}$ Gynaecologic and Obstetrics Clinic, Department of Women's and Children's Health, University of Padua, Padua, Italy \\ ${ }^{5}$ Department of Obstetrics and Gynecology, “Filippo Del Ponte” Hospital, University of Insubria, Varese, Italy
}

\begin{abstract}
Stress urinary incontinence (SUI) is defined as a complaint of inadvertent loss of urine occurring as a result of an increase in intraabdominal pressure. Strong evidence supports the use of pelvic floor muscle training (PFMT) as the first-line conservative treatment for SUI. Extracorporeal magnetic stimulation (EMS) is a noninvasive, effective, acceptable, and safe therapeutic modality for SUI. Although PFMT and EMS share most of their influences on the pathophysiology of SUI, it is unclear whether one of these routinely used treatment modalities is superior to another in terms of improvement of clinical outcomes or cost-effectiveness. To the best of our knowledge, no randomized controlled trials have so far directly compared PFMT with EMS. Our aim here is to describe a protocol for such a study.

This will be a parallel-group, single-blind, randomised controlled trial compliant with the SPIRIT, CONSORT, and TIDieR reporting guidelines. Participants will be women aged 18 to 65 years who have previously given at least one vaginal delivery (at least 12 months before joining the study) who present with symptoms of SUI lasting at least 6 months yet have not previously received treatment for it. In the first study arm, patients will receive an eight-week, high-intensity, home-based Kegel exercises regimen. In the second study arm, the treatment scheme will consist of 2 sessions of EMS per week for a total of eight weeks. The primary outcome will be effectiveness of treatment as measured by the International Consultation on Incontinence Questionnaire Urinary IncontinenceShort Form overall score, eight weeks, three months, and six months after commencement of treatment.
\end{abstract}

Key words: stress urinary incontinence, extracorporeal magnetic stimulation, pelvic floor, pelvic muscle.

\section{Introduction}

Stress urinary incontinence (SUI) is defined as a complaint of inadvertent loss of urine occurring as a result of an increase in intraabdominal pressure, in the absence of a detrusor muscle contraction [1]. Estimates of the prevalence vary widely in the published literature, due in part to differing terminologies, diagnostic approaches, and populations studied [1-3]. It has been reported that SUI affects up to $14 \%$ of premenopausal women and up to $35 \%$ of postmenopausal women [4, 5], unfavourably affecting quality of life (QoL), sexual function, and mental health [6, 7]. Pathophysiologically, SUI is caused by urethral deficiency and impairment of supportive structures such as the levator ani muscle, endopelvic fascia, and pubourethral ligament [8].
The beneficial effect of pelvic floor muscle training (PFMT) on female urinary incontinence and pelvic organ prolapse was first described in 1948 by Arnold Kegel, who reported an astonishing success rate of $84 \%$ [9]. More recent studies have demonstrated somewhat lower success rates of PFMT than those reported by Kegel, but still in the impressive range from 56 to $75 \%$ based on improved clinical outcomes [10]. Strong evidence and grade $A$ recommendations support the use of PFMT as the first-line conservative treatment for SUI [11]. Although alternative exercise regimens for SUI have been investigated, their clinical implementation is not yet recommended [12].

Other conservative therapeutic options for SUI include vaginal cones, bladder training, as well as elec-

\section{Corresponding author:}

Giovanni Buzzaccarini, MD, Gynaecologic and Obstetrics Clinic, Department of Women's and Children's

Submitted: 19.08 .202

Health, University of Padua, via Nicolò Giustiniani 3, 35128, Padua, Italy,

Accepted: 13.09 .2021

e-mail: giovanni.buzzaccarini@gmail.com 
trical or magnetic stimulation. Extracorporeal magnetic stimulation (EMS) is a non-invasive, effective, acceptable, and safe therapeutic modality for SUI $[6,7]$. In EMS, induction of a changing magnetic field results in a flow of electrons within the field, controlled depolarisation of the adjacent nerves, and subsequent muscular contraction [13]. Although the optimal frequency and duration of treatment are not yet established, findings of a systematic review have suggested that higher frequency and pulse duration provide an adequate modality for achieving treatment efficacy [14]. At our clinic, which is the largest Croatian urogynaecological referral centre, EMS is routinely offered as an alternative to women who are not willing to perform Kegel exercises. Considering that a direct comparison of these two common conservative methods is lacking, we decided to perform a randomized controlled trial comparing the efficacy of PFMT versus EMS for the treatment of SUI. In this paper we describe the protocol of the study.

\section{Material and methods}

\section{Study design}

This is a clinical trial protocol. In particular, we will perform a parallel group, single-blind, randomised controlled trial compliant with the SPIRIT, CONSORT and TIDieR reporting guidelines. The study will be conducted at the Department of Obstetrics and Gynaecology, Clinical Hospital Centre Zagreb, Croatia. The trial was registered at clinicaltrials.gov Identifier: NCT04307680 on $13^{\text {th }}$ March 2020.

\section{Recruitment of participants}

Participants will be women aged 18 to 65 years who have previously given at least one vaginal delivery (at least 12 months before joining the study), who present with symptoms of SUI lasting at least 6 months and without previous treatment for urinary incontinence. All women attending our urogynaecological outpatient clinic with a referral diagnosis of suspected urinary incontinence will undergo an initial examination by a physician specialised in urogynaecology. This screening will include:

- physical examination including checking of bulbocavernosus reflex,

- blood glucose level assessment with a glucometer,

- pregnancy test (for premenopausal women),

- a gynaecological examination with prolapse staging using the pelvic organ prolapse-qualification (POP-Q),

- pelvic floor muscle function assessment by digital palpation and a perineometer,

- International Consultation on Incontinence Questionnaire Urinary Incontinence-Short Form (ICIQ-UISF) assessment.
According to the International Urogynecological Association a(IUGA) guidelines, urinalysis by urine dipstick is highly recommended as screening for urinary tract infection [15]. Based on the findings from a urine dipstick and patient's symptomatology, we will determine whether to perform a urine culture (mid-stream). The final diagnosis of SUI, in accordance with our standardised diagnostic protocol which has been adopted from the IUGA guidelines [15], will be made at the end of the initial examination by the attending physician. The diagnostic specific criterion of SUI is the observation of involuntary leakage from the urethra, synchronous with exertion, sneezing or coughing [15].

All patients with diagnosis of SUI will be asked to participate in the trial. After acceptance patients will be randomized according to the treatment arm (Group 1_PMFT and Group 2_EMS) and will be required to complete the following questionnaires: ICIQ-UI-SF; the Urogenital Distress Inventory (UDI-6); the International Consultation on Incontinence Questionnaire Lower Urinary Tract Symptoms Quality of Life Module (ICIQLUTSqol). All questionnaires will be completed at enrolment (T0), at the end of the treatment (T1, 8 weeks) and during the follow-up period (T2 three months after treatment and T3 six months after treatment). The Patient Global Impression of Improvement (PGI-I) questionnaire will be completed at T1, T2 and T3.

A schematic diagram of the participant timeline is available in Table 1.

\section{Inclusion and exclusion criteria}

Women will be offered participation if they meet the following conditions:

- between 18 and 65 years old,

- instantaneous leakage with cough stress test or positive cough stress test at a bladder volume of $200-250 \mathrm{ml}$,

- history of vaginal childbirth (not more recent than 12 months ago),

- symptoms of SUI for at least 6 months,

- ICIQ-UI-SF score of at least 6 points at the initial visit. Exclusion criteria will be the following:

- pregnant women,

- women actively trying to conceive,

- cognitive impairment or intellectual disability,

- neurological disorders (e.g. epilepsy, Parkinson disease, multiple sclerosis, cerebrovascular insult),

- lack of independent mobility and polytraumatic patients,

- active vulvovaginitis,

- vulvodynia,

- ongoing urinary tract infection,

- any form of prior conservative treatment for SUI,

- previous surgery for SUI,

- history of pelvic irradiation,

- type III SUI based on the Blaivas clinical classification, 
Table 1. Time schedule of enrolment, interventions, assessments, and visits for participants

\begin{tabular}{|c|c|c|c|c|c|c|}
\hline TIMEPOINT & $\begin{array}{l}\text { Pre-study } \\
\text { screening, } \\
\text { enrolment } \\
\text { and consent }\end{array}$ & $\begin{array}{l}\text { Baseline/ } \\
\text { randomisation } \\
\text { and allocation }\end{array}$ & $\begin{array}{c}\text { T0 } \\
\text { prior to } \\
\text { treatment }\end{array}$ & $\begin{array}{c}\text { T1 } \\
\text { after } \\
\text { treatment }\end{array}$ & $\begin{array}{c}\text { T2 } \\
3 \text { months } \\
\text { after end } \\
\text { of treatment }\end{array}$ & $\begin{array}{c}\text { T3 } \\
6 \text { months } \\
\text { after end } \\
\text { of treatment }\end{array}$ \\
\hline \multicolumn{7}{|l|}{ Enrolment } \\
\hline Eligibility screen & $x$ & & & & & \\
\hline Informed consent & $x$ & & & & & \\
\hline Allocation & & $x$ & & & & \\
\hline \multicolumn{7}{|l|}{ Interventions } \\
\hline PFMT & & & 8 weeks & & & \\
\hline EMI & & & 8 weeks & & & \\
\hline \multicolumn{7}{|l|}{ Assessments } \\
\hline Baseline variables & $x$ & $x$ & & & & \\
\hline $\begin{array}{l}\text { Primary outcome (ICIQ-UI-SF } \\
\text { overall score) }\end{array}$ & & & & $x$ & & \\
\hline Secondary outcome variables & & & $x$ & $x$ & $x$ & $x$ \\
\hline PGI-I scale assessment & & & & $x$ & $x$ & $x$ \\
\hline PFMT & & & $x$ & $x$ & & \\
\hline
\end{tabular}

PFMT - pelvic floor muscle training, EMI - extracorporeal magnetic innervation, ICIQ-UI-SF - International Consultation on Incontinence Questionnaire Urinary Incontinence-Short Form, PGI-I - impressionPatient Global Impression of Improvement

- stage 2 or higher according to POP-Q classification,

- implanted metallic devices, such as cardiac pacemaker or urethral stent,

- patients in treatment with digoxin,

- present use of medications that are known to affect continence mechanisms (e.g. $\alpha$-adrenergic antagonists, diuretics, serotonin-norepinephrine reuptake inhibitors),

- random blood glucose level above $10 \mathrm{mmol} / \mathrm{l}$

- inability to contract the pelvic floor muscles,

- other subtypes of urinary incontinence, diagnosed by symptom assessment, genitourinary examination, or ultrasound,

- suspected urethral/vesical fistula,

- postvoid residual volume greater than $200 \mathrm{ml}$.

- allergy to latex,

- not understanding Croatian,

- lack of informed consent.

\section{Interventions}

After randomization, we will include the participants in two arms.

\section{Pelvic floor muscle training Group 1}

In the first study arm, patients will receive an 8-week, high-intensity, home-based Kegel exercises regimen in order to sufficiently increase strength, endurance, and coordination of muscle activity. Patients will be initially instructed to perform 5 fast (3 seconds) contractions and 10 sustained (no time limit) contractions, with 10-second relaxation periods in between, three times a day. The first week target is 200 successful contrac- tions ( $1: 2$ ratio between fast and sustained contraction), whereas in each successive week the overall number of contractions should be increased by $10 \%$. Patients will in addition be instructed to use pelvic muscle contractions for urge inhibition and preventive contractions with exertional events such as coughing, sneezing, or lifting. A description of the PFMT programme will be sent by a nursing assistant to participants by e-mail, after initial enrolment as well as during the 8-week intervention period, tailored by the attending physician. No additional interventions to enhance PFMT effectiveness will be provided.

\section{Extracorporeal magnetic innervation Group 2}

Extracorporeal magnetic stimulation employs powerful pulsing magnetic fields to stimulate nerve activity in the pelvic floor, which in turn exercises the muscles that control bladder function, improving strength and endurance as well as increasing circulation. The treatment scheme will consist of 2 sessions per week for 8 weeks in total. Each session will last 30 minutes, consisting of a 15-min low frequency stimulation programme $(10 \mathrm{~Hz})$ followed by 15 minutes of high-frequency stimulation programme $(50 \mathrm{~Hz})$. The intensity of EMS will be adjusted to the maximum level tolerable by the patient and will be immediately discontinued if any adverse effect is reported by the patient. Both allocated interventions will be discontinued on participant request.

\section{Measurements}

Women who accept participation in the study and sign informed consent will be asked to complete specific questionnaires. Moreover, pelvic floor muscle function will be evaluated using a perineometer. 
International Consultation on Incontinence Questionnaire-Urinary Incontinence Short Form, UDI-6, ICIQ-LUTSqol will be completed at enrolment (TO), at the end of the treatment (T1, 8 weeks) and during the follow-up period ( $\mathrm{T} 2$ three months after treatment and T3 six months after treatment). The Patient Global Impression of Improvement Questionnaire questionnairewill be completed at T1, T2 and T3. The pelvic floor muscle function evaluated by a perineometer will be performed at T0 and T1.

\section{Perineometer}

The perineometer is a non-invasive device, which quantifies the maximum voluntary contraction using a vaginal probe to capture alterations in intra-vaginal pressure, measured in $\mathrm{CmH}_{2} \mathrm{O}$. Three maximum contractions are requested from the participant with rest intervals of 30 seconds, and the highest value achieved is recorded. In order to exclude the influence of intra-abdominal pressure, only contractions with evident inward perineal movement will be considered valid. The vaginal probe is labelled at a distance of $3.5 \mathrm{~cm}$ before every measurement, in order to obtain equal probe placement and to minimize measurement bias.

\section{Patient reported outcome measures}

The International Consultation on Incontinence Questionnaire-Urinary Incontinence Short Form

consists of three scored items related to the frequency of urination, amount of leakage, and impact on everyday life. The additional fourth item does not contribute to the total score, but rather aims to determine the type of urinary incontinence. The total score is the sum of the first three items, ranging from 0 to 21, with higher scores indicating greater severity of symptoms. The proposed critical values categorise the results as follows: $\leq 5=$ mild urinary incontinence, $6-12=$ moderate, $13-18$ = severe, $\geq 19$ = very severe [16].

The Urogenital Distress Inventory is a six-item inventory assessing symptoms associated with lower urinary tract dysfunction and genital prolapse. Item responses range from 0 "not at all" to 3 "greatly". The total score is obtained by averaging the results of all items multiplied by $331 / 3$ to put scores on a scale of 0 to 100 . Higher scores indicate more symptom distress. In addition to the total score, three subscales can be calculated: irritative symptom subscale ( $1^{\text {st }}$ and $2^{\text {nd }}$ item), stress symptom subscale ( $3^{\text {rd }}$ and $4^{\text {th }}$ item), and obstructive/ discomfort symptom subscale ( $5^{\text {th }}$ and $6^{\text {th }}$ item) [17].

The International Consultation on Incontinence Questionnaire Lower Urinary Tract Symptoms Quality of Life Module is a disease-specific questionnaire consisting of 20 items and providing information on the impact of the SUI on the patients' quality of life, with a particular focus on social aspects. It is intended for adult respondents and it has been translated into more than 20 languages.
Patient Global Impression of Improvement is a simple tool which allows patients to quantify their response to treatment and overall satisfaction level. There are seven possible answer categories ranging from very much better to very much worse and scored between 1 and 7.

Additional clinically relevant information will be collected using a 3-day bladder diary prior to and after treatment (T0 and T1, respectively), as well as 3 and 6 months after the last treatment session (T2 and T3, respectively). This information will be used to determine the dynamics in overall incontinence episodes in both study arms. Further information which will supplement our study will determine the proportion of women in each study arm who will perform PFMT after their assigned treatment is done.

\section{Other data}

Of all participants the following sociodemographic data will be collected: age, parity, delivery mode, contraception use, menopausal status, education level, job, disease, alcohol use, and body mass index (BMI).

\section{Outcomes}

The primary outcome will be the effectiveness of treatment as measured by the ICIQ-UI-SF overall score, eight weeks after commencement of treatment. Secondary outcomes will include:

the average increase in the vaginal pressure, as measured with the Peritron perineometer at eight weeks,

women's satisfaction with treatment, measured by the Patient Global Impression of Improvement (PGI-I) scale at $\mathrm{T} 1, \mathrm{~T} 2$ and $\mathrm{T} 3$,

symptom distress, using the UDI-6 and ICIQ-UI-SF questionnaire at the initial visit (TO), after the intervention is completed (T1), three months (T2) and six months (T3) following the end of the intervention,

quality of life, measured by ICIQ-LUTSqol (4-point reduction according to Nyström et al. is clinically relevant) also at the initial visit (TO), after the intervention is completed (T1), three months (T2) and six months (T3) following the end of the intervention [2].

\section{Power analysis}

The sample size was determined based on the primary outcome: the proportion of women who have responded to treatment, defined as at least a 4-point reduction in the overall ICIQ-UI-SF score. Because Kegel exercises will be unsupervised during the whole eight weeks of the intervention period, we conservatively estimated that $30 \%$ of women are going to respond in the PFMT group $[1,18,19]$. 
Assuming a $60 \%$ response in the EMS group [20, 21], a sample size of 80 women (40 per group) will be needed at the alpha level of 0.05 and statistical power of $80 \%$ to demonstrate superiority of EMS over PFMT. Allowing for attrition of $25 \%$, we will aim to recruit a total of 100 women (50 per arm). The computer program Clinstat was used for sample size calculation.

\section{Randomisation and allocation concealment}

An investigator with no clinical involvement in the trial used Clinstat to generate a random allocation sequence in random blocks of size two, three, four, or five in each group. After the research nurse obtains the woman's consent, she will telephone a researcher who is independent of the recruitment process for allocation assignment.

\section{Blinding}

Patients and interventionists will be aware of the allocated arm. Investigators and staff, including outcome assessors who are experienced clinicians, will be kept blind to assignment of the participants. All investigators, staff, and participants will be blinded to trial results until analyses are completed.

\section{Safety profile and adverse events}

Both tested interventions are established treatments for SUI and are considered safe. Serious adverse events are unlikely; however, participants will be monitored, and any potential adverse events recorded. Any reported adverse events will be assessed, with appropriate diagnostic and treatment procedures provided as needed.

\section{Dafa collection and management}

The data collection and management procedures have been approved by the Institutional Review Board and are in accordance with the general data protection regulation. Personal information about patients will be kept separate from the main dataset and will not be shared. All personal data will be stored securely in order to protect confidentiality before, during, and after the trial. Trained staff will conduct data entry and coding in the trial's database. Since there is no sponsorship or other competing interests, the interventions are short, and no serious adverse events are likely, the data monitoring committee was deemed unnecessary.

\section{Statistical methods}

Descriptive statistics measures (arithmetic mean, standard deviation, minimum and maximum values, and total range) will be used to show the distributions of the participants. Categorical variables will be displayed as frequencies with their percentages. In order to calculate whether there are statistically significant differences initially, immediately after the treatment, and 3 and 6 months after the treatment in ICIQ-UI-SF results between the two groups (EMS vs. PFMT), we will conduct mixed-model analysis of variance. Moreover, we will perform regression models analysis in order to adjust for confounders, especially age, menopausal status, BMI and the total number of vaginal deliveries. At each follow-up point we will record the number of dropouts. Analyses will be intention-to-treat. If necessary, we will calculate the impact of missing data by either sensitivity analyses or imputation techniques. A two-sided $p$-value $\leq 0.05$ will be considered statistically significant. Data analyses will be performed using SPSS 23.0 (IBM Corp., Armonk, NY).

\section{Ethics}

The study has been approved by the Institutional Review Board of the University Hospital Center Zagreb (No. 021-1/149-19). The study will adhere to all the principles of the Declaration of Helsinki and an additional approval will be sought from the Ethics Committee of the University of Zagreb School of Medicine.

Only women who provide written consent after full verbal and written information about the purpose of the trial as well as its potential risks and benefits will be able to participate. All participants will be able to withdraw at any time without consequences and without stating reasons for further non-participation. Only the investigators will have access to the dataset, which will be password-protected.

\section{Discussion}

To our knowledge, PFMT and EMT have not been previously compared directly in a randomised controlled trial. Management of SUI is based mainly on available conservative treatment modalities [15]. Likewise, a recent meta-analysis concluded that there is Level 1 , Grade A evidence of the effectiveness of PFMT [1]. Although the mid-urethral sling procedure is considered the gold standard treatment for SUI, it may not be suitable for all patients, particularly in women with significant comorbidities, which are common in older age [21]. Moreover, it can lead to postoperative voiding dysfunction, including urine retention in up to $36 \%$ of patients [22]. Other, non-specific complications such as postoperative pain, wound infections and mesh erosion may permanently impair QoL [23].

Most available conservative treatment approaches are designed to improve the function of pelvic floor muscles in order to maintain positive urethral closure 
pressure during an increase of intra-abdominal pressure. Other approaches are based on behavioural techniques and lifestyle modification, such as smoking cessation, dietary change, and subsequent weight loss [4]. There is also a dependence on a synergy between the pelvic floor structures and adjacent nervous systems which is essential for establishing biofeedback therapy [24].

Because there is no standardised PFMT treatment programme, various estimates of success have been reported between studies [23-25]. Precisely, PFMT is associated mostly with symptom improvements, and its short-term cure rate is up to $80 \%$, defined as $<2 \mathrm{~g}$ of leakage on different pad tests [26]. Nonetheless, there is no consensus on the optimal duration of PFMT, which varied between 4 and 12 weeks in previous trials [25]. Supervised intensive PFMT obtains the best results in risk reduction of those patients who remain incontinent $[18,27]$. However, in a randomised study by Felicíssimo et al., which compared supervised PFMT with unsupervised PFMT, no difference was found in pelvic floor muscle strength, ICIQ-UI-SF score, pad test, weekly exercise compliance, or subjective evaluation of urinary loss [19].

In our proposed study protocol, we have decided to include an 8-week, high-intensity, home-based regimen in order to sufficiently increase strength, endurance, and coordination of muscle activity and to achieve an optimal overall patient treatment response. Our training regimen is a modification of the one previously proposed by Wyman et al., involving concurrently three components, i.e. strength, endurance, and coordination [28]. To facilitate compliance, we set the initial goal to a minimum of 100 contractions per week, which can be gradually increased by $5-10 \%$ on a weekly basis, depending on participant determination. We believe that the proposed PFMT duration of 8 weeks can provide sufficient progress and provide further encouragement for practice prolongation at a home as well as work setting.

Extracorporeal magnetic stimulation presents an alternative approach in providing pelvic floor muscle enhancement. The mechanism of action is based on the controlled depolarisation of the adjacent nerves and subsequent muscular contraction. In clinical practice, EMS draws attention chiefly because of its high acceptance and tolerability rates among patients, non-invasiveness, and high long-term participation rates [6, 14, 21]. Moreover, improved QoL scores and minimal adverse effects suggest an attractive non-surgical alternative to patients with SUI who are not candidates for surgical management [21]. However, information about the effectiveness and safety of EMS mostly comes from single-arm studies, which are prone to bias [14]. Another limitation in widespread clinical practice is the lack of an established definition of frequency and duration of treatment. Systematic reviews suggest higher frequen- cy and pulse duration as an adequate modality in order to achieve treatment efficacy [14].

In a randomised, double-blind, sham-controlled study involving 120 female subjects with SUI, the response rate after 8 weeks (16 sessions overall) was $75 \%$, compared to $22 \%$ in the sham-controlled group [21]. However, in this study, the primary response criterion was a 5 -point reduction in the overall ICIQ-UI-SF score, which represents a greater margin compared to our primary outcome, which is based on Nyström et al. $[2,21]$. It is reasonable to assume that reported EMS success rates in their study [21] could be even greater if criteria for conservative as opposed to surgical management were applied. In our study protocol, we plan to use a 3-point reduction threshold, as no other study has provided information on how a change in the ICIQ-UI-SF questionnaire scores can be interpreted clinically [2]. However, we will attempt to establish the minimal important difference in our study population correlating the ICIQ-questionnaires with the PGI-I.

According to a systematic review and meta-analysis of randomised controlled trials that studied conservative management of SUI, evidence of direct comparison between PFMT and EMS is lacking [23]. Most published reports including EMS were sham-controlled trials while incorporation of PFMT was missing $[6,14,23]$. This highlights the need for conducting the present study.

In order to further inform the interpretation of outcomes during follow-up, we will assess the proportion of women in either study arm who will perform PFMT following the 8-week intervention period. Although PGI-I will be used primarily to assess patient satisfaction, it will in addition provide indirect information on adherence to treatment in both study arms.

All patients treated for SUI at our centre routinely receive a multidisciplinary lifestyle intervention, which includes dietary counselling, in order to reduce bodyweight. This might contribute to improvements in both study arms. In a non-blinded prospective interventional study, with a mean weight loss of $3.6 \mathrm{~kg}$ participants reported a significant improvement in QoL [3].

Reflecting on the diagnostic procedures which will be used in our proposed study, we believe that an invasive diagnostic approach (e.g. urodynamic evaluation) is avoidable. Although some authors argue that it can provide depth to the understanding of overall SUI pathophysiology, urodynamic evaluation is invasive, relatively expensive, and does not affect clinical decision-making [15]. In a study by Norton et al., it was estimated that in women with uncomplicated SUI, up to 33 million US dollars could be saved annually by not performing urodynamic evaluation [29].

In order to overcome subjectivity in digital palpation measurements, we will use the perineometer as an objective and validated tool to verify pelvic floor muscle strength at several time points: prior to (TO) and after 
(T1) treatment, as well as 3 (T2) and 6 (T3) months after the final treatment session [30]. However, we adopted and modified a scheme of three maximum voluntary pelvic floor muscle contractions with a prolonged rest period of 30 seconds instead of the previously reported 10 -second rest interval $[30,31]$. This will provide a more practical yet still a reasonable and sufficient interval for muscle recovery between contractions. Published reports about perineometer reliability are encouraging, although there is low concordance between the measurements obtained with the devices of different manufacturers [30, 32]. Consequently, it is important to achieve a consensus on which device will be used to facilitate reproducibility and comparison in future research [33]. Considering a recent cost-utility analysis of nine different surgical interventions [34], a similar cost-effectiveness study on conservative SUI management using Markov microsimulation modelling may be warranted. A further area of future research may be to develop more accurate instruments for measurement of treatment adherence.

\section{Conclusions}

Our randomised trial will provide the evidence from a direct comparison of two common conservative treatments for SUI, using state-of-the-art clinical and research methodologies. The results will directly inform clinical decision making in caring for patients with SUI.

\section{Acknowledgement}

Ethical aspects of this randomised controlled trial include approval from the Institutional Review Board and the Ethics Committee of the University of Zagreb School of Medicine. The study will adhere to all the principles of the Declaration of Helsinki. The results will be published in peer-reviewed academic journals and disseminated to patient organisations and media. All patients will sign an informed consent form.

\section{Disclosure}

The authors report no conflict of interest.

\section{References}

1. Nie X-F, Ouyang Y-Q, Wang L, et al. A meta-analysis of pelvic floor muscle training for the treatment of urinary incontinence. Int J Gynecol Obstet 2017; 138: 250-255.

2. Nyström E, Sjöström $M$, Stenlund $H$, et al. ICIQ symptom and quality of life instruments measure clinically relevant improvements in women with stress urinary incontinence: ICIQ-UI SF and ICIQ-LUTSqol Correlation to PGI-I. Neurourol Urodyn 2015; 34: 747-751.

3. Fjerbæk A, Søndergaard L, Andreasen J, et al. Treatment of urinary incontinence in overweight women by a multidisciplinary lifestyle intervention. Arch Gynecol Obstet 2020; 301: 525-532.
4. Luber KM. The definition, prevalence, and risk factors for stress urinary incontinence. Rev Urol 2004; 6 Suppl 3: S3-9.

5. Hunskaar S, Lose $G$, Sykes $D$, et al. The prevalence of urinary incontinence in women in four European countries. BJU Int 2004; 93: 324-330.

6. Lim R, Liong ML, Leong WS, et al. Patients' perception and satisfaction with pulsed magnetic stimulation for treatment of female stress urinary incontinence. Int Urogynecology J 2018; 29: 997-1004.

7. Peng L, Zeng X, Shen $\mathrm{H}$, et al. Magnetic stimulation for female patients with stress urinary incontinence, a meta-analysis of studies with shortterm follow-up. Medicine (Baltimore) 2019; 98: e15572.

8. McGuire EJ. Pathophysiology of stress urinary incontinence. Rev Urol 2004; 6 Suppl 5: S11-S17.

9. Kegel $\mathrm{AH}$. Progressive resistance exercise in the functional restoration of the perineal muscles. Am J Obstet Gynecol 1948; 56: 238-248.

10. Chevalier F, Fernandez-Lao C, Cuesta-Vargas Al. Normal reference values of strength in pelvic floor muscle of women: a descriptive and inferential study. BMC Womens Health 2014; 14: 143.

11. Abrams P, Andersson KE, Birder L, et al. Fourth international consultation on incontinence recommendations of the international scientific committee: Evaluation and treatment of urinary incontinence, pelvic organ prolapse, and fecal incontinence. Neurourol Urodyn 2010; 29: 213-240.

12. B $\varnothing K$, Herbert RD. There is not yet strong evidence that exercise regimens other than pelvic floor muscle training can reduce stress urinary incontinence in women: a systematic review. J Physiother 2013; 59: 159-168.

13. Quek P. A critical review on magnetic stimulation: what is its role in the management of pelvic floor disorders? Curr Opin Urol 2005; 15 : 231-235.

14. Lim R, Lee SWH, Tan PY, et al. Efficacy of electromagnetic therapy for urinary incontinence: a systematic review: efficacy of electromagnetic therapy in urinary incontinence. Neurourol Urodyn 2015; 34: 713-722.

15. Ghoniem G, Stanford E, Kenton K, et al. Evaluation and outcome measures in the treatment of female urinary stress incontinence: international urogynecological association (IUGA) guidelines for research and clinical practice. Int Urogynecology J 2007; 19: 5-33.

16. Avery K, Donovan J, Peters TJ, et al. ICIQ: a brief and robust measure for evaluating the symptoms and impact of urinary incontinence. Neurourol Urodyn 2004; 23: 322-330.

17. Uebersax JS, Wyman JF, Shumaker SA, et al. Short forms to assess life quality and symptom distress for urinary incontinence in women: the incontinence impact questionnaire and the urogenital distress inventory. Neurourol Urodyn 1995; 14: 131-139.

18. Hay-Smith EJC, Herderschee R, Dumoulin C, et al. Comparisons of approaches to pelvic floor muscle training for urinary incontinence in women. Cochrane Database Syst Rev Published Online First: 7 December 2011.

19. Felicíssimo MF, Carneiro MM, Saleme CS, et al. Intensive supervised versus unsupervised pelvic floor muscle training for the treatment of stress urinary incontinence: a randomized comparative trial. Int Urogynecology J 2010; 21: 835-840.

20. Yamanishi T, Suzuki T, Sato R, et al. Effects of magnetic stimulation on urodynamic stress incontinence refractory to pelvic floor muscle training in a randomized sham-controlled study. LUTS 2019; 11: 61-65.

21. Lim R, Liong ML, Leong WS, et al. Pulsed magnetic stimulation for stress urinary incontinence: 1-year followup results. J Urol 2017; 197: 13021308.

22. Van Leijsen SAL, Kluivers KB, Mol BWJ, et al. Value of urodynamics before stress urinary incontinence surgery: a randomized controlled trial. Obstet Gynecol 2013; 121: 999-1008.

23. Moroni R, Magnani P, Haddad J, et al. Conservative Treatment of Stress Urinary Incontinence: a systematic review with meta-analysis of randomized controlled trials. RBGO Gynecol Obstet 2016; 38: 097-111.

24. Krishna Dass A, Lo T-S, Khanuengkitkong S, et al. Diagnosis and conservative management of female stress urinary incontinence. Gynecol Minim Invasive Ther 2013; 2: 48-51.

25. Ayeleke RO, Hay-Smith EJC, Omar MI. Pelvic floor muscle training added to another active treatment versus the same active treatment alone for urinary incontinence in women. In: The Cochrane Collaboration, ed. Cochrane Database of Systematic Reviews, John Wiley \& Sons Ltd., Chichester, UK 2013. 
26. Bø K. Pelvic floor muscle training in treatment of female stress urinary incontinence, pelvic organ prolapse and sexual dysfunction. World J Urol 2012; 30: 437-443.

27. Dumoulin C, Hay-Smith J, Habée-Séguin GM, et al. Pelvic floor muscle training versus no treatment, or inactive control treatments, for urinary incontinence in women: a short version Cochrane systematic review with meta-analysis: pelvic floor muscle training versus no treatment for urinary incontinence in women. Neurourol Urodyn 2015; 34: 300-308.

28. Wyman JF, Fantl JA, McClish DK, et al. Comparative efficacy of behavioral interventions in the management of female urinary incontinence. Am J Obstet Gynecol 1998; 179: 999-1007.

29. Norton PA, Nager CW, Brubaker L, et al. The cost of preoperative urodynamics: a secondary analysis of the ValUE trial: cost of preoperative urodynamics. Neurourol Urodyn 2016; 35: 81-84.

30. Ribeiro J dos S, Guirro EC de O, Franco M de M, et al. Inter-rater reliability study of the PeritronTM perineometer in pregnant women. Physiother Theory Pract 2016; 32: 209-217.

31. Gilling PJ, Wilson LC, Westenberg AM, et al. A double-blind randomized controlled trial of electromagnetic stimulation of the pelvic floor vs. sham therapy in the treatment of women with stress urinary incontinence. BJU Int 2009; 103: 1386-1390.

32. Rahmani N, Mohseni-Bandpei MA. Application of perineometer in the assessment of pelvic floor muscle strength and endurance: A reliability study. J Bodyw Mov Ther 2011; 15: 209-214.

33. Jasięga J, Jaszczak E. The problem of urinary incontinence among wom en aged 20-40. Physiother Rev 2019; 23.

34. Javanbakht M, Moloney E, Brazzelli M, et al. Economic evaluation of surgical treatments for women with stress urinary incontinence: a costutility and value of information analysis. BMJ Open 2020; 10: e035555. 